\title{
Reliability Testing on the Printed Circuit Board of Mobile Phone using Infrared Thermography
}

by Hoon Joo(1), Won-Tae Kim(2), Man-Yong Choi(3)

(1) IR Wave Corp., Seoul, Korea

(2) Dept. of Bio-mechanical Engineering, Kongju National University, Chungnam, Korea, 340-702

(3) Life Measurement Group, Korea Research Institute of Standards and Science, Daejeon, Korea, 305-600

\begin{abstract}
In this paper, reliability testing using infrared thermography is performed to investigate the failure and abnormal sympton of mobile phone in operation. As roles of the presented work, IR system for the analysis of PCB reliabilty was set-up to be appled as nondestructive testing method. From IR thermographic images obtained, following results are reviewed. First, prior confirmation and forecast for troble points by intensive control for the IR thermal profile. Secondly, available aging test with long time under real temperature, Thirdly, by making database for past results, it was possible the history control and worth for comparison data. Also, using IR thermographic images detected already, audits for mixed board are analyzed under several operational signals. From this work, it was assumed that IR testing is capable of saving the test time and increasing the test accuracy by diverse costomized functions.
\end{abstract}

Keywords: Reliability Testing, Infrared(IR) Thermography, Nondestructive Testing(NDT), Mobile Phone, Thermal Profile 